\title{
Less SAD with more sun and serotonin
}

DOI:

10.1038/nrn2263
Short, dark winter days put most of us in a gloomy mood, but in people with seasonal affective disorder (SAD), they can cause severe clinical depression. Fortunately, this depression can be treated with bright-light therapy (BLT), and it disappears altogether in summer. Willeit et al. now show that these changes in mood are associated with alterations in the efficiency of the serotonin (5-hydroxytryptamine) transporter (5-HTT) in the patients' blood platelets.

One theory of depression posits that impaired functioning of monoamine neurotransmitters, such as serotonin, causes the disorder, but it is unknown how this impairment might arise. Serotonin levels in the synapse are controlled by the 5-HTT, and Willeit and colleagues therefore investigated whether alterations in 5-HTT functioning might underlie depression in SAD.

The authors compared people with SAD with healthy volunteers, and assessed 5-HTT functioning in winter, after 4 weeks of BLT and in summer. They did this by measuring 5-HTT-mediated inward and outward transport in blood platelets (which are easily obtainable). In winter, both inward transport rate and outward transport were enhanced in the platelets of SAD patients compared with healthy controls. Importantly, these differences in platelet 5-HTT functioning disappeared after 4 weeks of BLT and were absent in summer. The number of 5-HTTs and their affinity for serotonin did not change with BLT or with the seasons, indicating that the increased 5-HTT inward transport that was found in SAD patients was due to increased efficiency of the transporter.

The authors also assessed the patients' depression levels at the three time points, using a structured interview. They found that posttreatment, both inward transport rate and outward transport correlated with depression scores in SAD patients. Moreover, patients whose depression did not decrease after treatment did not show a change in 5-HTT-mediated outward transport after treatment.

Patients and healthy controls were genotyped for variations in the promoter region of the gene that encodes the 5-HTT, which have been associated with differential vulnerability to depression. In this study, the distribution of the genotypes did not differ between SAD patients and healthy people, and the genotype did not influence serotonin transport efficiency.

This study showed that changes in 5-HTT efficiency parallel those in depression scores, and that an antidepressant treatment which does not pharmacologically target 5-HTTs nevertheless induces alterations in the transport mechanism. If the efficiency of platelet 5-HTTs reflects that of transporters in the brain, the findings suggest that an increase in 5-HTT efficiency might contribute to low synaptic serotonin levels in people with depression.

Leonie Welberg

\section{ORIGINAL RESEARCH PAPER}

Willeit, M. et al. Enhanced serotonin transporter function during depression in seasonal affective disorder. Neuropsychopharmacology 19 Sep 2007 (doi:10.1038/sj.npp.1301560)

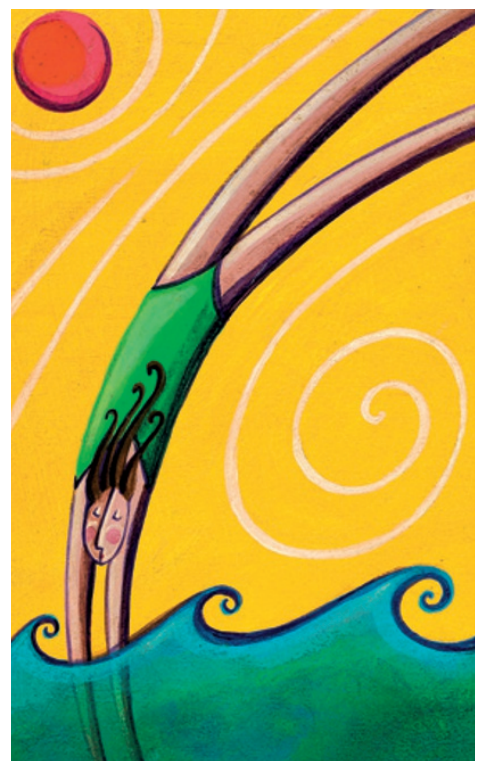

